\title{
Progression from Unilateral to Bilateral Parkinsonism in Early Parkinson Disease: Implication of Mesocortical Dopamine Dysfunction by PET
}

Shunsuke Yagi ${ }^{1}$, Etsuji Yoshikawa ${ }^{2}$, Masami Futatsubashi ${ }^{2}$, Masamichi Yokokura $^{3}$, Yujiro Yoshihara ${ }^{3}$, Tatsuo Torizuka ${ }^{4}$, and Yasuomi Ouchi ${ }^{1}$

${ }^{1}$ Molecular Imaging Frontier Research Center, Hamamatsu University School of Medicine, Hamamatsu, Japan; ${ }^{2}$ Central Research Laboratory, Hamamatsu Photonics K.K., Hamamatsu, Japan; ${ }^{3}$ Department of Psychiatry and Neurology, Hamamatsu University School of Medicine, Hamamatsu, Japan; and ${ }^{4}$ Research Center for Child Mental Development, Hamamatsu University School of Medicine, Hamamatsu, Japan

It is still unclear why some early Parkinson disease (PD) patients with unilateral parkinsonism develop bilateral parkinsonism soon after the diagnosis is made as Hoehn and Yahr (HY) stage 1 and others remain stable for a long time. Here, we examined in vivo changes in the brain dopaminergic system using PET with a dopamine transporter radiotracer, ${ }^{11} \mathrm{C}-2-\mathrm{B}$-carbomethoxy-3B-(4-fluorophenyl) tropane $\left({ }^{11} \mathrm{C}-\mathrm{CFT}\right)$, to elucidate the pathophysiologic characteristics of the dopamine system in early converters. Methods: Twelve drug-naïve PD patients with HY stage 1 disease and 8 age-matched healthy subjects participated in this study. Clinical evaluation of their parkinsonism was performed monthly until their HY stage 1 (unilateral parkinsonism) disease had become stage 2 (bilateral parkinsonism) disease according to the Unified Parkinson Disease Rating Scale. The endpoint of the follow-up study was the time of the conversion. Region-of-interest analysis was used to examine ${ }^{11} \mathrm{C}$-CFT binding in the mesocortical (nucleus accumbens, caudate, orbitofrontal cortex) and nigrostriatal (putamen) dopamine projection regions. Multiregression analyses between these PET data and clinical parameters were performed within the PD group. Results: Between-group comparisons showed that, irrespective of the duration of conversion, all PD patients clinically diagnosed at $\mathrm{HY}$ stage 1 had a significant reduction in ${ }^{11} \mathrm{C}-\mathrm{CFT}$ binding in the bilateral striatum (affected, $-46 \%$; unaffected, $-35 \%)$. Regression analysis showed that the level of ${ }^{11} \mathrm{C}-\mathrm{CFT}$ binding in the nucleus accumbens and orbitofrontal cortex on the unaffected side was significantly positively correlated with the conversion interval. This positive correlation indicates that the more severe a dysfunction presents in the mesocortical dopamine system on the seemingly intact side, the more rapidly the parkinsonism proceeds to the intact side (bilateral parkinsonism). Conclusion: The finding of bilateral reduction in the striatal ${ }^{11} \mathrm{C}-\mathrm{CFT}$ binding even in $\mathrm{HY}$ stage 1 PD patients confirms that molecular changes in the dopamine

Received Mar. 1, 2010; revision accepted Apr. 28, 2010.

For correspondence or reprints contact: Yasuomi Ouchi, Molecular Imaging Frontier Research Center, Hamamatsu University School of Medicine, 1-20-1 Handayama, Higashi-ku, Hamamatsu 431-3192, Japan. E-mail: ouchi@hama-med.ac.jp

COPYRIGHT $\odot 2010$ by the Society of Nuclear Medicine, Inc. system precede clinical phenotype, suggesting an advantage of PET for detecting an early abnormality of the disease. The spread of parkinsonism to the unaffected side soon after the diagnosis of HY stage 1 PD may be related to the degree of mesocortical dopamine dysfunction.

Key Words: ${ }^{11} \mathrm{C}-\mathrm{CFT}$; mesocortical dopamine function; unilateral parkinsonism; Parkinson disease; positron emission tomography

J Nucl Med 2010; 51:1250-1257

DOI: 10.2967/jnumed.110.076802

$\mathbf{T}$ he diagnosis of Parkinson disease (PD) is based on clinical assessment, which is also used to evaluate disease progression before therapeutic intervention. The neuropathologic process involved in PD is loss of the nigral dopamine neurons and the ensuing loss of dopamine nerve terminals in the striatum $(1,2)$. Although the extent of the annual reduction in dopamine neurons is estimated to be around $10 \%(3,4)$, the exact rate of disease progression varies from patient to patient. This variation makes decisions on medication difficult in the clinical setting because administering the normal doses of levodopa and other antiparkinsonian drugs irrespective of the severity of parkinsonian symptoms would not always be appropriate. We previously reported that even in early-stage PD, dopamine dysfunction is present not only in the striatal but also in the mesocortical dopaminergic projection system and that mesocortical dysfunction might contribute to mental and behavioral impairment (5). Our findings indicate that the level of dopamine function in the mesocortical system may be a key surrogate marker of the progression of PD.

Many in vivo studies have used PET with several radiotracers for presynaptically located dopamine transporters to 
match PET findings with pathology in the postmortem PD brain $(6,7)$. The advantage of using PET is its ability to depict functional abnormalities in dopamine neurons at the molecular level before clinical phenotypes appear. For example, motor symptoms do not develop until 50\%-60\% of dopamine neurons (binding of tracers) have been affected $(8,9)$. Although PET of the dopamine transporter cannot differentiate the number of dopamine transporters per synapse from the number of dopamine terminals in PD, imaging of the dopamine transporter is of diagnostic value because reduction in dopamine transporter comes earlier than changes in dopamine content in the PD-model monkeys (10). Therefore, this technique allows us to predict the progression of the disease by examining the vulnerability of dopamine neurons at the molecular level.

The purpose of the present study was to compare the binding level of ${ }^{11} \mathrm{C}$-2-B-carbomethoxy-3B-(4-fluorophenyl) tropane $\left({ }^{11} \mathrm{C}-\mathrm{CFT}\right.$, a marker for the membrane dopamine transporter) in dopaminergic projection (mesocortical and nigrostriatal) regions at an early stage of PD (Hoehn and Yahr [HY] stage 1) with the interval of conversion from HY stage 1 to HY stage 2 to clarify whether dopamine abnormalities in any brain region can act as a surrogate marker of the clinical progression of early-stage PD.

\section{MATERIALS AND METHODS}

\section{Participants}

We studied 12 drug-naïve patients with HY stage $1 \mathrm{PD}$ ( 5 men, 7 women; mean age $\pm \mathrm{SD}, 58.8 \pm 9.3 \mathrm{y})$ and 8 healthy subjects ( 5 men, 3 women; mean age \pm SD, $51 \pm 15.5 \mathrm{y})$. We obtained their written informed consent for the present study, which was also approved by the local ethics committee of Hamamatsu Medical Center. Each PD patient was clinically assessed using the Unified Parkinson Disease Rating Scale (UPDRS). Patients who had limb tremors, rigidity, and bradykinesia and who had received a tentative clinical diagnosis of PD were recruited from our main hospital or the neighboring clinics. Controls who were physically healthy and did not have or were not being treated for any neurologic complications were recruited by inhouse advertisement. All participants underwent MRI, a neuropsychiatric examination, and a blood test to exclude the possibility of any accompanying disease. None of the participants had major depression, dementia, or a history of psychostimulant drug abuse. The UPDRS evaluation was performed just before the PET examination. The UPDRS scores varied from 11 to 25 . The duration of disease was from 4 to 33 mo (mean, $16.2 \mathrm{mo}$ ). The disease duration was defined by the time from initial symptoms. Because all PD patients were diagnosed as having a clinical severity of HY stage 1 , their limbs were affected unilaterally (left-sided in 4 patients and right-sided in 8 patients). More detailed clinical characteristics of the patients are listed in Table 1.

\section{MRI and PET}

Before the PET scan, MRI (0.3-T MRP7000AD; Hitachi) was performed with 3-dimensional mode sampling (repetition time/echo time, 200/23; flip angle, $75^{\circ}$; slice thickness, $2 \mathrm{~mm}$, with no gap; matrices, $256 \times 256$ ). Each piece of MRI data was used to determine suitable areas for region-of-interest (ROI) analysis. With the aid of data relating to tilt angle and spatial coordinates obtained during the procedure for determining the intercommisural (anterior commissure-posterior commissure [AC-PC]) line on each subject's sagittal MR images, a PET gantry was set parallel to the AC-PC line by tilting and moving the gantry for each study. This set-up enabled us to reconstruct the PET images parallel to the AC-PC line, without reslicing (11).

The details of the PET procedure are given elsewhere $(5,12)$. In brief, we used a high-resolution brain PET scanner with 24 detector rings yielding 47 slice images simultaneously (SHR12000; Hamamatsu Photonics KK). After head-fixation using a thermoplastic face mask and a 10min transmission scan for attenuation correction, serial scans (time frames, $4 \times 30,20 \times 60$, and $14 \times 300 \mathrm{~s}$ ) were

\section{TABLE 1. Characteristics of PD Patients}

\begin{tabular}{rlcccll} 
Patient no. & Sex & Age $(\mathrm{y})$ & Duration of illness $(\mathrm{mo})^{\star}$ & UPDRS total & Affected side & $\begin{array}{l}\text { Initial symptom } \\
\text { Rigidity }\end{array}$ \\
\hline 1 & F & 44 & 18 & $11(2 / 3 / 6)$ & $\mathrm{L}$ & Rigidity/tremor (UL/LL) \\
2 & $\mathrm{~F}$ & 55 & 12 & $13(2 / 3 / 8)$ & $\mathrm{L}$ & Tremor (UL) \\
3 & $\mathrm{M}$ & 67 & 14 & $14(1 / 2 / 11)$ & $\mathrm{R}$ & Tremor (UL/LL) \\
4 & $\mathrm{~F}$ & 60 & 24 & $16(2 / 3 / 11)$ & $\mathrm{R}$ & Rigidity/tremor (UL) \\
5 & $\mathrm{M}$ & 58 & 25 & $16(4 / 6 / 6)$ & $\mathrm{R}$ & Rigidity/tremor (UL) \\
6 & $\mathrm{~F}$ & 58 & 10 & $17(2 / 5 / 10)$ & $\mathrm{R}$ & Rigidity/tremor (UL) \\
7 & $\mathrm{~F}$ & 71 & 9 & $18(2 / 4 / 12)$ & $\mathrm{R}$ & Rigidity/tremor (UL/LL) \\
8 & $\mathrm{M}$ & 56 & 33 & $18(4 / 4 / 10)$ & $\mathrm{R}$ & Rigidity/tremor (UL/LL) \\
9 & $\mathrm{~F}$ & 73 & 4 & $20(2 / 2 / 16)$ & $\mathrm{L}$ & Rigidity \\
10 & $\mathrm{M}$ & 64 & 12 & $21(2 / 4 / 15)$ & $\mathrm{R}$ & Rigidity/tremor (UL) \\
11 & $\mathrm{M}$ & 43 & 28 & $21(2 / 6 / 13)$ & $\mathrm{L}$ & Rigidity/tremor (UL) \\
12 & $\mathrm{~F}$ & 57 & & $25(4 / 5 / 16)$ & $\mathrm{R}$ &
\end{tabular}

*Duration between disease onset and PET examination.

UPDRS scores in parentheses are mentation/activities of daily living/motor examination.

$\mathrm{UL}=$ upper limb; $\mathrm{LL}=$ lower limb. 
obtained for $92 \mathrm{~min}$ after a slow bolus venous injection of a $6 \mathrm{MBq} / \mathrm{kg}$ dose of ${ }^{11} \mathrm{C}$-CFT. Arterial blood sampling was not performed in the present study.

\section{Image Data Processing}

Irregular ROIs were located bilaterally on the nucleus accumbens, nucleus caudate, ventral putamen, dorsal putamen, orbitofrontal cortex, and cerebellum (Fig. 1) on the MR images (13). The ROIs were then automatically transferred onto the corresponding ${ }^{11} \mathrm{C}$-CFT distribution images (reconstructed from 70 to $90 \mathrm{~min}$ after the tracer injection) using image-processing software (Dr View; Asahi Kasei Co.) on a workstation (Hypersparc ss-20; SUN Microsystems) (5). We calculated the reference tissue-derived ratio index (RI) (i.e., the ratio of the PET count in the target region to the PET count in the cerebellum in the late integrated image) because the value of this ratio reflects the binding potential estimated by the quantitative 3-compartment 4-parameter model for ${ }^{11} \mathrm{C}$-CFT, which we tested previously (14). The PET image used for the ROI setting comprised 2 consecutive images that covered slices (thickness, $6.8 \mathrm{~mm}$ ) in the $z$ direction (15), so each ROI value contained functional information about the striatum
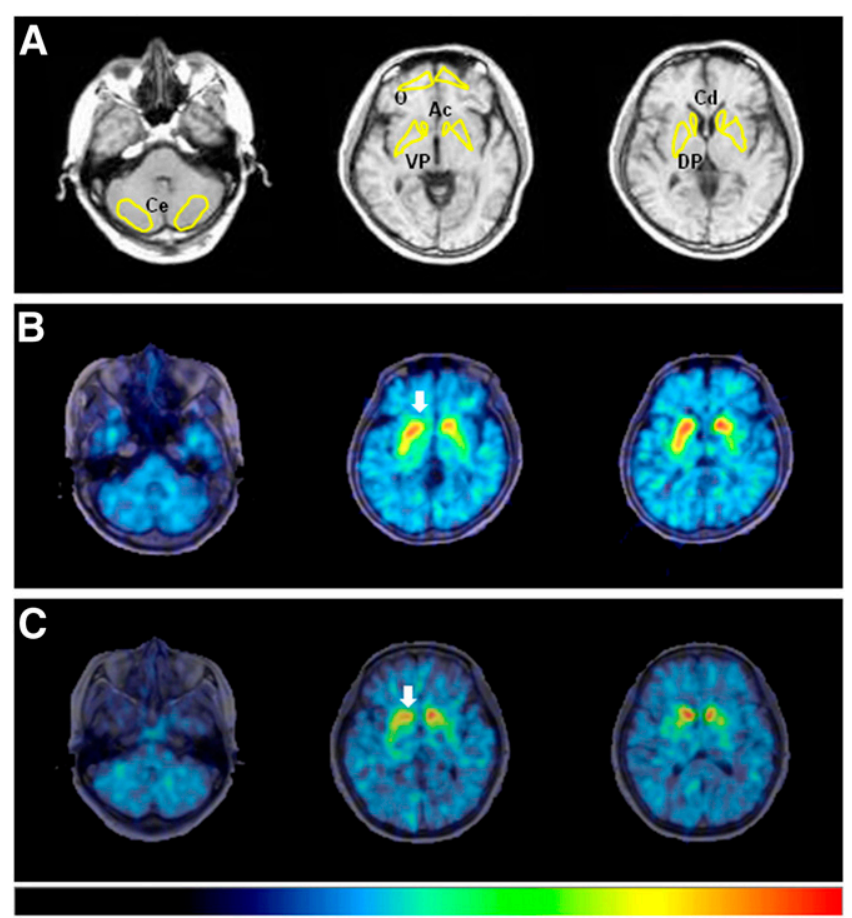

FIGURE 1. ${ }^{11} \mathrm{C}-\mathrm{CFT}$ PET images and ROI setting. (A) Irregular ROIs, drawn bilaterally on concerned regions on MR images, were placed on corresponding PET images. (B) PD patient with longer conversion period from $\mathrm{HY}$ stage 1 to $\mathrm{HY}$ stage 2. (C) PD patient with shorter conversion period from $\mathrm{HY}$ stage 1 to $\mathrm{HY}$ stage 2. Arrow indicates reduction in ${ }^{11} \mathrm{C}-\mathrm{CFT}$ binding in nucleus accumbens. Color bar indicates quantified level of RI (from 0 to 3$)$. Ac = nucleus accumbens; $\mathrm{Cd}=$ caudate; $\mathrm{Ce}=$ cerebellum; $\mathrm{DP}=$ dorsal putamen; $\mathrm{O}=$ orbitofrontal cortex; VP = ventral putamen. to a depth of at least $6.8 \mathrm{~mm}$ in the $z$ direction (volume data).

\section{Statistics}

Student $t$ tests were conducted to compare all estimates in the brain regions between the PD and control groups. A $P$ value of less than 0.05 was considered to be statistically significant. Although an age-related reduction in ${ }^{11} \mathrm{C}-\mathrm{CFT}$ binding was reported (16), we compared the estimates without age correction because there was no difference in age between the 2 groups. Pearson regression coefficient analysis was performed for comparisons between ${ }^{11} \mathrm{C}-\mathrm{CFT} \mathrm{RI}$ values and the patients' clinical variables. To examine dopamine projection regions in which alterations in ${ }^{11} \mathrm{C}-\mathrm{CFT}$ binding might be linked to disease prognosis, multiple regression analyses were performed between the regional ${ }^{11} \mathrm{C}$-CFT levels at HY stage 1 and the duration required for the conversion from HY stage 1 to HY stage 2. A $P$ level of less than 0.05 after Bonferroni adjustment was used to indicate statistical significance.

\section{RESULTS}

\section{Level of ${ }^{11} \mathrm{C}-\mathrm{CFT}$ Binding in HY Stage $1 \mathrm{PD}$}

Compared with the values in their healthy counterparts (Table 2), ${ }^{11} \mathrm{C}$-CFT RI values in the PD patients were significantly reduced not only on the affected side but also on the unaffected side. The following were the reduced values in the different regions: ventral putamen, $50 \%$ on the affected side versus $39 \%$ on the unaffected side; dorsal putamen, $61 \%$ on the affected side versus $45 \%$ on the unaffected side; nucleus accumbens, $38 \%$ on the affected side versus $32 \%$ on the unaffected side; caudate, $34 \%$ on the affected side versus $22 \%$ on the unaffected side; and orbitofrontal cortex, $34 \%$ on the affected side versus $28 \%$ on the unaffected side.

\section{Correlation Between ${ }^{11} \mathrm{C}-\mathrm{CFT}$ Binding and Clinical Variables at HY Stage 1}

Statistics with Bonferroni adjustment showed that all regression results fell outside the statistically significant limit (Fig. 2) ( $P>0.05$, corrected for multiple comparison). The ${ }^{11} \mathrm{C}$-CFT RI values in each brain region tended to be negatively correlated with the UPDRS scores: nucleus accumbens (affected: $r=-0.408$; unaffected: $r=-0.787$ ), caudate (affected: $r=-0.709$; unaffected: $r=-0.703$; $P>0.05$ corrected), orbitofrontal cortex (affected: $r=$ -0.404; unaffected: $r=-0.58$ ); ventral putamen (affected: $r=-0.603$; unaffected: $r=-0.455)$; dorsal putamen (affected: $r=-0.682$; unaffected: $r=-0.564$ ). There was also a tendency toward a negative correlation between the UPDRS subscores and the ${ }^{11} \mathrm{C}$-CFT RI values (not shown).

\section{Association of Initial ${ }^{11} \mathrm{C}-\mathrm{CFT}$ Binding with Clinical Progression}

As shown in Table 3, all PD patients were treated with antiparkinsonian drugs, among which levodopa was 


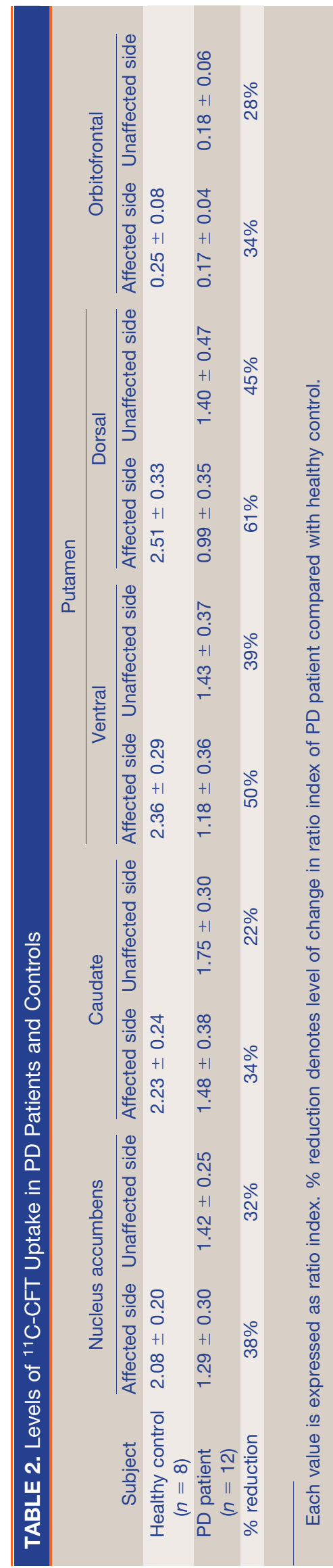

prescribed to all patients. Only 1 patient who had undergone a PET examination dropped out, because of a family issue. The mean duration required for conversion from $\mathrm{HY}$ stage 1 to stage 2 was $2.9 \pm 1.5 \mathrm{y}$ and ranged from 6 mo to $5 \mathrm{y}$ in the PD group. No specific relevance was found between the conversion interval and the kinds of antiparkinsonian drugs administered, and the doses of each drug varied mildly among the patients.

Regression analyses with Bonferroni adjustment showed that the initial values of ${ }^{11} \mathrm{C}-\mathrm{CFT} \mathrm{RI}$ in the nucleus accumbens, caudate, and orbitofrontal cortex on the unaffected side were significantly positively correlated with the conversion interval (nucleus accumbens, affected: $r=0.628$, $P>0.05$, unaffected: $r=0.889, P<0.05$ corrected; caudate, affected: $r=0.709, P>0.05$, unaffected: $r=$ $0.866, P<0.05$ corrected; and orbitofrontal cortex, affected: $r=0.682, P>0.05$, unaffected: $r=0.735$, $P<0.05$ corrected) (Fig. 3). In other words, dopamine hypofunction in the mesocortical dopamine system contralateral to the affected limb likely indicates rapid progression to bilateral parkinsonism. There was a tendency toward a positive correlation in these regions on the affected side (ventral putamen, affected: $r=0.695$, unaffected: $r=0.685$; dorsal putamen, affected: $r=0.510$, unaffected: $r=0.689$ ).

\section{DISCUSSION}

The diagnosis of PD and determining its prognosis are difficult in the clinical setting, as shown by previous studies that reported that the initial diagnoses of PD made by general neurologists were found to be incorrect at autopsy in $24 \%-35 \%$ of cases $(17,18)$. In addition, around $8.1 \%$ of the patients diagnosed with PD were later found to have an alternate diagnosis based on multifactorial clinical diagnostic criteria after a mean follow-up of 6 y (19). These reports indicate the difficulty of making a precise evaluation of possible PD cases based solely on clinical data. In contrast, depicting abnormalities of the dopamine system on a molecular basis has a greater advantage $(6,20-25)$. Previous studies showed that the progression of PD was associated with a similar rate of dopaminergic losses in all striatal subregions (26). In line with the results of previous studies, our findings (initial dopaminergic loss in the posterodorsal striatum and the following mesocortical dopamine dysfunction) are best interpreted as evidence of colinearity of degeneration, with progression across striatal regions. In other words, patients with initially more significant denervation (yet clinically still in HY stage 1) were likely to reach the conversion point sooner than were stage 1 patients with less severe denervation. This might be true because the correlation coefficients across the striatal subregions all showed an $r$ value greater than 0.5 . The higher levels of statistical significance for the anterior striatal regions is better explained by relative floor denervation effects in the more posterior regions (27). 
FIGURE 2. Correlations between UPDRS score at $\mathrm{HY}$ stage 1 and ${ }^{11} \mathrm{C}$ CFT RI values. Dashed lines for $\bigcirc$ and chain lines for $\boldsymbol{\Delta}$ show all tendencies of negative correlations. $\bigcirc=$ affected side; $\boldsymbol{\Delta}=$ unaffected side.

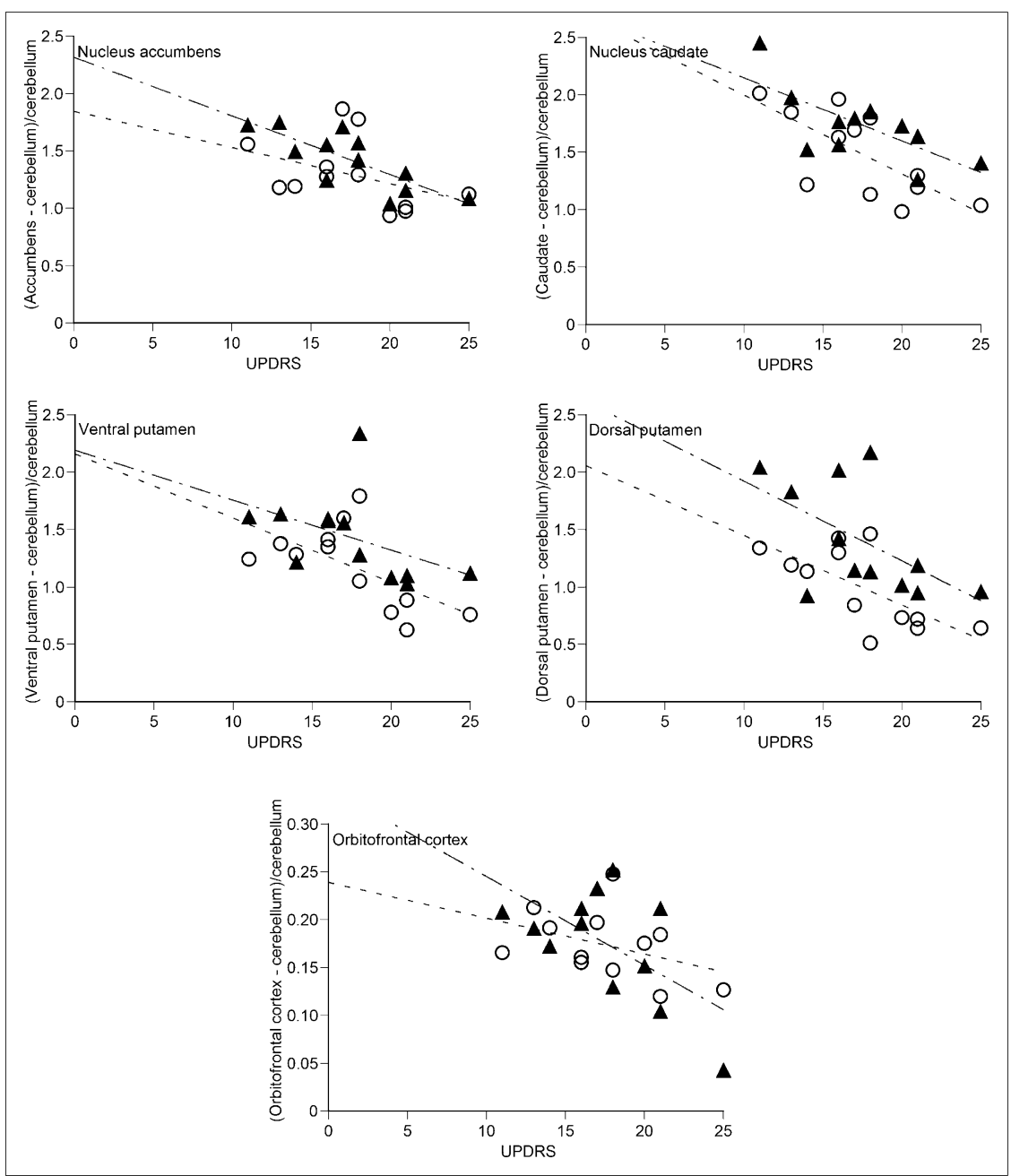

In all patients with $\mathrm{HY}$ stage $1 \mathrm{PD}$, a significant reduction in ${ }^{11} \mathrm{C}$-CFT binding was found in the dorsal putamen, which was in line with the previous findings from early PD patients with HY stage 1 or stage 2 disease (6).
This reduction is also consistent with pathologic evidence showing the most severe neuronal loss in the ventrolateral part of the substantia nigra, which projects mainly to the posterior putamen $(9,28)$. However, this regional

\section{TABLE 3. Details of PD Patients at Time of Progression to HY Stage 2}

\begin{tabular}{|c|c|c|c|c|c|c|}
\hline $\begin{array}{c}\text { Patient } \\
\text { no. }\end{array}$ & $\begin{array}{l}\text { Interval } \\
(y)^{*}\end{array}$ & $\begin{array}{l}\% \text { Increase } \\
\text { of UPDRS }{ }^{\dagger}\end{array}$ & $\begin{array}{l}\text { Levodopa } \\
\text { (mg/d) }\end{array}$ & $\begin{array}{c}\text { Dopamine } \\
\text { agonist (mg/d) }\end{array}$ & $\begin{array}{l}\text { Anticholinergic } \\
\text { agent (mg/d) }\end{array}$ & $\begin{array}{l}\text { Selegiline } \\
\text { (mg/d) }\end{array}$ \\
\hline 1 & 5 & 73 & 200 & 0.5 & 4 & - \\
\hline 2 & 5 & 100 & 100 & 0.5 & 4 & - \\
\hline 3 & 2 & 71 & 200 & 0.75 & 4 & 5 \\
\hline 4 & 3 & 38 & 100 & 0.5 & 4 & - \\
\hline 5 & 1.8 & 75 & 200 & 0.75 & 4 & 5 \\
\hline 6 & 4 & 65 & 100 & 0.5 & 4 & - \\
\hline 7 & 4.4 & 33 & 100 & 0.5 & 4 & - \\
\hline 8 & 3 & 33 & 100 & 0.5 & 4 & - \\
\hline 9 & 2 & 40 & 100 & 0.5 & 4 & - \\
\hline 10 & - & - & - & - & - & - \\
\hline 11 & 1.5 & 48 & 200 & 0.75 & 4 & 5 \\
\hline 12 & 0.5 & 44 & 200 & 0.75 & 4 & 5 \\
\hline
\end{tabular}




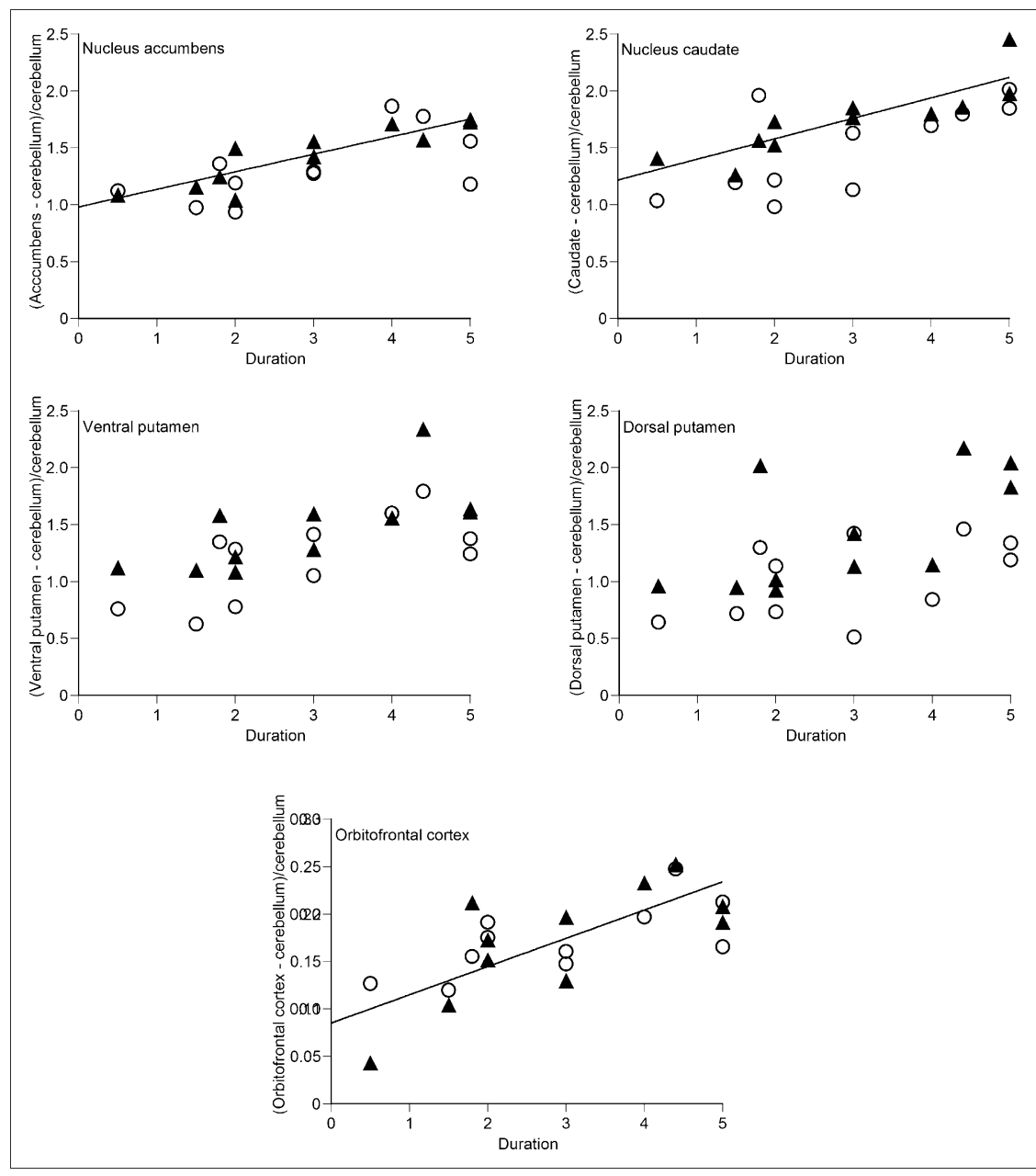

FIGURE 3. Correlations between conversion interval $(\mathrm{y})$ and ${ }^{11} \mathrm{C}-\mathrm{CFT} \mathrm{RI}$ values. Straight lines for $\boldsymbol{\Delta}$ show significant correlations $(P<0.05)$. $\bigcirc=$ affected side; $\boldsymbol{\Delta}=$ unaffected side. vulnerability does not always result in an orderly progression of parkinsonism-that is, a prolonged period at $\mathrm{HY}$ stage 1 or quick conversion from stage 1 to stage 2 , and such, in the clinical setting. In our study, we examined the correlation between the clinical progression of PD (HY stage 1 to stage 2 ) and regional ${ }^{11} \mathrm{C}-\mathrm{CFT}$ binding. As a result, we found that the reduction of ${ }^{11} \mathrm{C}$-CFT binding in the dorsal putamen did not correlate significantly with conversion time (progression), irrespective of its laterality. In contrast, ${ }^{11} \mathrm{C}-\mathrm{CFT}$ binding in the nucleus accumbens, caudate, and orbitofrontal cortex on the unaffected side was significantly positively associated with conversion time, suggesting that mesocortical dopamine function determines the prognosis of the disease. ${ }^{11} \mathrm{C}-\mathrm{CFT}$ binding in the mesocortical dopamine region did not correlate with symptomatic deterioration (data not shown). Rather, nigrostriatal dysfunction may reflect a symptomatologic change in PD, as reported elsewhere (29). Thus, mesocortical involvement may be an important predictor of the progression of PD from unilateral to bilateral parkinsonism. This in vivo finding is of biologic value because there is no clear consensus on whether the Braak pathologic progression theory (30) of PD has any clinical relevance (31).
Although all patients examined in the present study were diagnosed with unilateral parkinsonism (HY stage 1), striatal ${ }^{11} \mathrm{C}-\mathrm{CFT}$ binding was already significantly lower, even on the side ipsilateral to the affected limb. This observation was in line with a previous finding from a ${ }^{123} \mathrm{I}-\beta-\mathrm{CIT}$ SPECT study (32), supporting the authors' conclusion that imaging of dopamine tracer binding might serve as a tool for identifying individuals developing dopaminergic pathologic conditions before the onset of motor symptoms. Lateralization of parkinsonian symptoms with bilateral biotracer reduction in the striatum may be a common phenomenon at the time when patients first consult a doctor. There is no clear explanation as to why mesocortical dysfunction on the unaffected side leads to bilateral parkinsonism. One possibility is that any damage of the extrastriatal cortical region, especially the mesocortical projection region, would enhance latent further losses of putaminal dopamine transporter, which cause clinical manifestation of parkinsonism. Psychophysiologically, a dysfunction in the mesocortical dopamine system-which mediates affect, behavior, and cognition (33)—would negatively affect the nigrostriatal system through mutual neural interactions (34). Methodologically, however, one caveat is a low den- 
sity of dopamine transporter outside the striatal region (35). Despite this, the presence of different values in the orbitofrontal cortex could reflect a minor but significant alteration in PD pathophysiology.

In the present study, there was no marked difference in the medication administered during the course of the disease because the treatment of all patients was based on a standardized algorithm $(36,37)$. As a result, we could not clarify the differences between various treatment regimens. Although the dose of levodopa is similar among the patients in the current study, there would be a possibility that levodopa treatment might affect the parkinsonism progression. Because it has been recently reported that levodopa significantly affects the corticoputaminal loop but does not affect the corticocaudatal loop as much (38), the treatment might not be a significant confounding factor in the present result on the mesocortical system. Regarding antiparkinsonian drugs, there are reports that the progressive reduction in dopamine transporter binding was smaller during agonistbased therapy than in levodopa-based therapy (39) and that the mean improvement in total, motor, and ADL UPDRS scores was greater in the levodopa group. Antiparkinsonian drugs are considered to have some neuroprotective efficacy, but no established evidence by neuroimaging techniques has been reported in a clinical setting (40). Taken together, on the basis of our results, a cognitive stimulator that acts on the mesocortical dopamine system might be a promising drug treatment for HY stage 1 PD patients.

\section{CONCLUSION}

Because drug-naïve PD patients with unilateral parkinsonism diagnosed as HY stage 1 showed significantly lower levels of ${ }^{11} \mathrm{C}$-CFT binding in the bilateral striatum in the present study, it is clear that molecule-based dopamine functional alterations in the brain precede the PD phenotype. The progression of parkinsonism may possibly be related to additional dysfunction of the mesocortical dopamine system, although this dysfunction is of little use for predicting deterioration in extrapyramidal symptoms. Thus, molecular imaging with ${ }^{11} \mathrm{C}-\mathrm{CFT}$ is a useful method for assessing the pathophysiology of PD and its progression. Still, the mechanism of the development of PD remains unclear, as does the relevance of mesocortical neuronal damage to PD progression. Further, human-based imaging studies using other neurotransmitter tracers or a new tracer specific to the disease entity such as synuclein are needed.

\section{ACKNOWLEDGMENTS}

We thank Dr. Masanobu Sakamoto and Toshihiko Kanno and Yasuo Tanizaki (Hamamatsu Medical Center), Yutaka Naito (Japan Environment Research Corporation), and Akihito Oda (Hamamatsu Photonics KK) for their support. This work was supported by a Research Grant for Longevity Science from the Ministry of Health, Labor and Welfare, Japan, and a grant from the Takeda Science Foundation.

\section{REFERENCES}

1. German DC, Manaye K, Smith W, Woodward D, Saper C. Midbrain dopaminergic cell loss in Parkinson's disease: computer visualization. Ann Neurol. 1989;26:507-514.

2. McGeer PL, Itagaki S, Akiyama H, McGeer E. Rate of cell death in parkinsonism indicates active neuropathological process. Ann Neurol. 1988;24: 574-576.

3. Marek K, Innis R, van Dyck C, et al. $\left.{ }^{123} \mathrm{I}\right] \beta$-CIT SPECT imaging assessment of the rate of Parkinson's disease progression. Neurology. 2001;57:2089-2094.

4. Hilker R, Schweitzer K, Coburger S, et al. Nonlinear progression of Parkinson disease as determined by serial positron emission tomographic imaging of striatal fluorodopa F18 activity. Arch Neurol. 2005;62:378-382.

5. Ouchi Y, Yoshikawa E, Okada H, et al. Alterations in binding site density of dopamine transporter in the striatum, orbitofrontal cortex, and amygdala in early Parkinson's disease: compartment analysis for $\beta$-CFT binding with positron emission tomography. Ann Neurol. 1999;45:601-610.

6. Frost JJ, Rosier A, Reich S, et al. Positron emission tomographic imaging of the dopamine transporter with ${ }^{11} \mathrm{C}$-WIN 35,428 reveals marked declines in mild Parkinson's disease. Ann Neurol. 1993;34:423-431.

7. Frey KA, Koeppe R, Kilbourn M, et al. Presynaptic monoaminergic vesicles in Parkinson's disease and normal aging. Ann Neurol. 1996;40:873-884.

8. Fearnley JM, Lees AJ. Ageing and Parkinson's disease: substantia nigra regional selectivity. Brain. 1991;114:2283-2301.

9. Morrish PK, Rakshi JS, Bailey DL, Sawle GV, Brooks DJ. Measuring the rate of progression and estimating the preclinical period of Parkinson's disease with $\left[{ }^{18}\right.$ F]dopa PET. J Neurol Neurosurg Psychiatry. 1998;64:314-319.

10. Bezard E, Dovero S, Prunier C, et al. Relationship between the appearance of symptoms and the level of nigrostriatal degeneration in a progressive 1-methyl4-phenyl-1, 2, 3, 6-tetrahydropyridine-lesioned macaque model of Parkinson's disease. J Neurosci. 2001;21:6853-6861.

11. Ouchi Y, Nobezawa S, Okada H, Yoshikawa E, Futatsubashi M, Kaneko M. Altered glucose metabolism in the hippocampal head in memory impairment. Neurology. 1998;51:136-142.

12. Ouchi Y, Yoshikawa E, Sekine Y, et al. Microglial activation and dopamine terminal loss in early Parkinson's disease. Ann Neurol. 2005;57:168-175.

13. Mai J, Assheuer J, Paxinos G. Atlas of the Human Brain. New York, NY: Academic Press; 1997.

14. Ouchi Y, Okada H, Yoshikawa E, Nobezawa S, Futatsubash M. Brain activation during maintenance of standing postures in humans. Brain. 1999; 122:329-338.

15. Watanabe M, Shimizu K, Omura T, et al. A new high-resolution PET scanner dedicated to brain research. IEEE Trans Nucl Sci. 2002;49:634-639.

16. Rinne J, Sahlberg N, Ruottinen H, Nagren K, Lehikoinen P. Striatal uptake of the dopamine reuptake ligand $\left[{ }^{11} \mathrm{C}\right]$ beta-CFT is reduced in Alzheimer's disease assessed by positron emission tomography. Neurology. 1998;50: $152-156$.

17. Hughes AJ, Daniel SE, Kilford L, Lees AJ. Accuracy of clinical diagnosis of idiopathic Parkinson's disease: a clinico-pathological study of 100 cases. J Neurol Neurosurg Psychiatry. 1992;55:181-184.

18. Rajput AH, Rozdilsky B, Rajput A. Accuracy of clinical diagnosis in parkinsonism: a prospective study. Can J Neurol Sci. 1991;18:275-278.

19. Jankovic J, Rajput AH, McDermott MP, Perl DP. The evolution of diagnosis in early Parkinson disease. Arch Neurol. 2000;57:369-372.

20. Marek K, Jennings D, Tamagnan G, Seibyl J. Biomarkers for Parkinson's disease: tools to assess Parkinson's disease onset and progression. Ann Neurol. 2008;64:S111-S121.

21. Rinne OJ, Nurmi E, Ruottinen HM, Bergman J, Eskola O, Solin O. $\left[{ }^{18}\right.$ F] FDOPA and $\left[{ }^{18} \mathrm{~F}\right] \mathrm{CFT}$ are both sensitive PET markers to detect presynaptic dopaminergic hypofunction in early Parkinson's disease. Synapse. 2001;40:193-200.

22. Wong DF, Yung B, Dannals RF, et al. In vivo imaging of baboon and human dopamine transporters by positron emission tomography using $\left[{ }^{11} \mathrm{C}\right]$ WIN 35,428. Synapse. 1993;15:130-142.

23. Hantraye P, Brownell AL, Elmaleh D, et al. Dopamine fiber detection by $\left[{ }^{11} \mathrm{C}\right]-$ CFT and PET in a primate model of parkinsonism. Neuroreport. 1992;3:265268.

24. Nirenberg MJ, Vaughan RA, Uhl GR, Kuhar MJ, Pickel VM. The dopamine transporter is localized to dendritic and axonal plasma membranes of nigrostriatal dopaminergic neurons. J Neurosci. 1996;16:436-447.

25. Mozley PD, Schneider JS, Acton PD, et al. Binding of [ $\left.{ }^{99 \mathrm{~m}} \mathrm{Tc}\right]$ TRODAT-1 to dopamine transporters in patients with Parkinson's disease and in healthy volunteers. J Nucl Med. 2000;41:584-589. 
26. Nandhagopal R, Kuramoto L, Schulzer M, et al. Longitudinal progression of sporadic Parkinson's disease: a multi-tracer positron emission tomography study. Brain. 2009;132:2970-2979.

27. Martin WR, Wieler M, Stoessl A, Schulzer M. Dihydrotetrabenazine positron emission tomography imaging in early, untreated Parkinson's disease. Ann Neurol. 2008;63:388-394.

28. Szabo J. Organization of the ascending striatal afferents in monkeys. J Comp Neurol. 1980;189:307-321.

29. Benamer HTS, Patterson J, Wyper DJ, Hadley DM, Macphee GJA, Grosset DG. Correlation of Parkinson's disease severity and duration with ${ }^{123}$ I-FP-CIT SPECT striatal uptake. Mov Disord. 2000;15:692-698.

30. Braak H, Del Tredici K, Rub U, de Vos RA, Jansen Steur EN, Braak E. Staging of brain pathology related to sporadic Parkinson's disease. Neurobiol Aging. 2003;24:197-211.

31. Lees AJ, Hardy J, Revesz T. Parkinson's disease. Lancet. 2009;373:20552066.

32. Marek KL, Seibyl JP, Zoghbi SS, et al. $\left[{ }^{123} \mathrm{I}\right]$ beta-CIT/SPECT imaging demonstrates bilateral loss of dopamine transporters in hemi-Parkinson's disease. Neurology. 1996;46:231-237.

33. Lieberman A. Depression in Parkinson's disease: a review. Acta Neurol Scand. 2006;113:1-8
34. Wise RA. Roles for nigrostriatal-not just mesocorticolimbic-dopamine in reward and addiction. Trends Neurosci. 2009;32:517-524.

35. Ito H Takahashi H, Arakawa R, Takano H, Suhara T. Normal database of dopaminergic neurotransmission system in human brain measured by positron emission tomography. Neuroimage. 2008;39:555-565.

36. Vingerhoets FJ, Schulzer M, Calne D, Snow B. Which clinical sign of Parkinson's disease best reflects the nigrostriatal lesion? Ann Neurol. 1997;41:58-64.

37. Pahwa R, Factor SA, Lyons KE, et al. Quality Standards Subcommittee of the American Academy of Neurology. Practice parameter: treatment of Parkinson disease with motor fluctuations and dyskinesia (an evidence-based review) report of the Quality Standards Subcommittee of the American Academy of Neurology. Neurology. 2006;66:983-995.

38. Jubault T, Monetta L, Straffella AP, et al. L-dopa medication in Parkinson's disease restores activity in the motor cortico-striatal loop but does not modify the cognitive network. PLOS ONE. 2009;4:e6154.

39. Parkinson Study Group. Dopamine transporter brain imaging to assess the effects of pramipexole vs levodopa on Parkinson disease progression. JAMA. 2002;287: 1653-1661.

40. Ravina B, Eidelberg D, Ahlskog JE, et al. The role of radiotracer imaging in Parkinson disease. Neurology. 2005;64:208-215. 